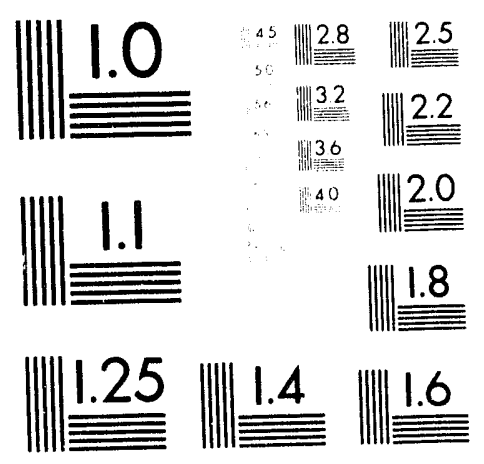



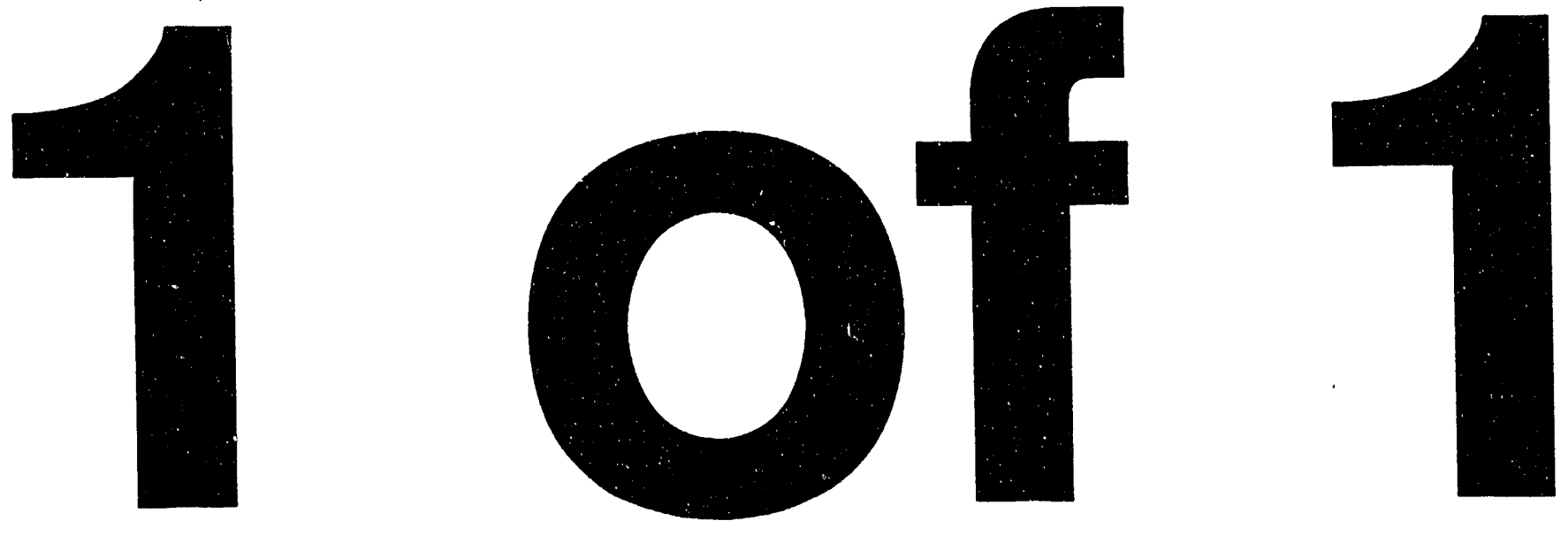


\author{
Model Calibration and Beam Control Systems for Storage Rings \\ W.J. Corbett ${ }^{*}$ M.J. Lee ${ }^{\dagger}$ and V. Ziemann ${ }^{\dagger}$ \\ Stanford Linear Accelerator Center \\ Stanford, CA 94309 USA
}

\begin{abstract}
Electron beam storage rings and linear accelerators are rapidly gaining worldwide popularity as scientific devices for the production of high-brightness synchrotron radiation. Today, everybody agrees that there is a premium on calibrating the storage ring model and determining errors in the machine as soon as possible after the beam is injected. In addition, the accurate optics model enables machine operators to predictably adjust key performance parameters, and allows reliable identification of new errors that occur during operation of the machine.

Since the need for model calibration and beam control systems is common to all storage rings, software packages should be made that are portable between different machines. In this paper, we report on work directed toward achieving in-situ calibration of the optics model, detection of alignment errors, and orbit control techniques, with an emphasis on developing a portable system incorporating these tools.
\end{abstract}

\title{
1.INTRODUCTION
}

Despite great care in the construction and installation of accelerator components, there are inevitably errors that can only be detected once the beam is injected into the storage ring. In most cases, the large errors are readily detected and corrected during the commissioning phase. For small errors, however, it is not uncommon to simply 'tune' the storage ring to optimize performance, but at the sarne time disregard the true source of error. Since the new '3rd generation' light sources (and most B-factory designs) are quite sensitive to optical and alignment errors, one must place increasingly more emphasis on error identification. An optics error, for example, generates ripple in the dispersion leading to resonant coupling between transverse and longitudinal particle oscillations at the accelerating cavities or collision point. Optics errors also modulate the cross-section of the beam which can reduce the radiation beam brightness or collider luminosity.

Magnet misalignments are damaging since they cause off-axis orbit trajectories through the non-linear fields of sextupole magnets, and steering errors at the synchrotron light source points. The off-axis sextupole fields lead to beam loss for particles executing complicated resonant trajectories. Steering errors require strong local corrections which can put the beam off-axis through undulator components or generate unwanted dispersion.

In order to detect and correct the source of these problems, we have developed algorithms for analyzing measurements of the corrector-to-beam position monitor (BPM) response matrix. To detect of optics errors, the most common approach is to deflect the beam with a transverse 'kick' and measure the difference in the beam trajectory. The single impulse kick is essentially a measurement of the Green's function of the accelerator, with the beam probing the spatial structure of the focusing components in the accelerator. The measured response is then compared to the simulated response calculated with the model values for the accelerator components. Discrepancies between model and measurement indicate error in the model of the as-built machine. The primary advantage gained by analysis of the 'difference orbit' data is that BPM offset values and quadrupole misalignments (dipole component) are subtracted away prior to the analysis. In section 2, we will discuss difference orbit analysis techniques, including simultaneous fitting of multiple trajectories to gain self-consistency of the result.

The problem of analyzing misalignments in the beamline is considerably more difficult. In the first place, one should always determine at least the first-order (quadrupole) optics model of the storage ring before attempting to detect zero-order (dipole) errors. This reduces the number of degrees of freedom in the problem. The problem is still complicated, however, since deflecting the beam through the effective dipole field of an offset quadrupole does not generate the same signature as the gradient field of the quadrupole. In addition, offset errors at the BPMs must be taken into account in any alignment analysis. It is therefore difficult to distinguish between quadrupole misalignments and BPM misalignments. Section 3 discusses several approaches that can be used to find the offset errors of components in a storage ring, and the difficulties of determining alignment errors based on measurements of the beam.

* research performed at SSRL which is operated by the Deparment of Energy, Office of Basic Sciences, Division of Chemical Sciences, Division of Materials Sciences.

† work supported by the Department of Energy Contract DE-AC03-76SF00515.

Contributed to SPIE's 1993 International Symposuim on Optics Imaging and Instumentation, San Diego, CA July 11-16,1993

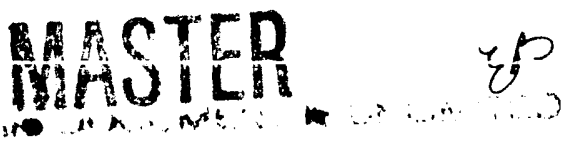


In section 4, orbit control algorithms are discussed with the emphasis on systems which can move the beam with minimum corrector strength.

In section 5, we discuss the need to develop integraced error analysis and beam control algorithms packaged so that the programs can be shared between laboratories. Sirice the investment in coding these rcutines large, and most laboratories use the same algorithms, the potential savings in effort is great. The goal is to develop a universal system with a standard protocol so that each new algorithm can be share 1 with laboratories around the world.

\section{MODEL CALIBRATION}

In this section, calibration of the optics model for circular accelerators is discussed. The exercise of calibration refers to developing a linear (or higher order) optics model for the as-built machine based on observation of the beam response to external perturbations. One first compares the measured data to numerical simulations obtained using the accelerator model, and where discrepancies occur, selected model parameters are used as fitting parameters to make the simulation agree with the measured data. Although non-linear properties of the lattice are included in any observation of the beam response, these effects are typically of lower magnitude than the quadrupole components. Hence, it is sensible to begin with calibration of the linear components before attempting to calibrate non-linear properties of the lattice.

\subsection{Response matrix measurement}

Most measurements of the transverse beam parameters are made either by perturbing quadrupole magnet strengths (to determine the local envelop modulation of the beam) or with dipole 'kick' perturbations to measure the 'response matrix' of the accelerator. The set of measurements of beam displacement at the BPMs due to all dipole kicks can be collected together to form the response matrix, or discretized Green's function, of the lattice. Since proper processing and interpretation of the response matrix data can lead to an accurate linear optics model for the accelerator, it is effective way to measure many accelerator properties; the Twiss parameters at all elements, the dispersion function, and the associated synchrotron integrals. Clearly, the response matrix measurement and its use for model calibration is a valuable diagnostic tool.

\subsection{Single-Lrack analysis}

The most simple way to analyze the response matrix is to work with the effect of single correctors on the beam displacement at all BPMs, that is, to concentrate on a single row of the response matrix. For this purpose, we have found the user interactive program RESOLVE [1] useful. RESOLVE has been used to detect modeling errors in PEP, SPEAR, the NSLS X-RAY ring, and many parts of the SLC, and to develop general methods for model calibration. As shown in Fig. 1, single-track analysis begins with the accelerator model containing numerical values of all components (strength and location of dipoles, quadrupoles, sextupoles, etc) the measured data in the desired row of the response matrix, and the associated corrector strength.

Next, one computes the closed orbit distortion (cod) due to the single corrector, as predicted by the accelerator model. If the agreement between the measured and model data is good, then the model accurately represents the as-built machine. Furthermore, the corrector strength has accurate conversion factors between setpoint and readback, and the BPM readings have accurate linearity. Pathological cases, where for instance the corrector strength is $10 \%$ high and the BPM linearities are all $10 \%$ low, are unlikely.

If the simulated closed orbit distorion does not agree with the measured data, then the fun begins. The typical recipe we use for detecting the source of error proceeds as follows:

Step 1. BPM Analysis - Look for isolated BPM readings that do not agree with the trend of the simulated trajectory. One fast way to determine bad monitors is to look in the plane transverse to the kick, where the displacement should be small for weakly coupled machines. If the suspect monitor reading is large in the transverse plane, the BPM reading is in error and eliminated from the fituing procedure.

Step 2. Corrector Analysis - Vary the corrector strength in the fitting procedure to improve the agreement between measure and simulated data. Now there is a single parameter (corrector strength) that scales the amplitude of the closed orbit to match the measured data. Often this step improves the quality of the fit, but is not the true solution to the problem. If a quadrupole power supply is out of calibration, for instance, the model tunes will not agree with the measured tune, and the closed orbit formula 


$$
x_{1}=\frac{\sqrt{\beta_{1} \beta_{1} \beta} \cos \left(v \pi-\left|\phi_{1}-\phi_{2}\right|\right)}{2 \sin v \pi},
$$

will be incorrect. $\left(x_{1}, \beta_{1}, \phi_{1}\right)$ and $\left(x_{2}, \beta_{2}, \phi_{2}\right)$ are the position, betafunction, and phase advance at the observation and kick positions, respectively. The sensitivity to the tune in the denominator can appear as a corrector mis-calibration.

Step 3. Quadrupole Family Errors - The quadrupole family strengths should also be used as fitting parameters. For the horizontal plane, one looks primarily for QF family errors (or $\mathrm{QD}$ in the vertical). The fitting of quadrupole families will generally bring the model tune into agreement with the measured tune. During this process, the variation on the quadrupole strengths away from the nominal value should be monitored to make sure the answers are physically viable. For measurements in the horizontal plane, the beam energy can also be varied to determine if there is a dispersion component in the orbit. Multi-track analysis should be used to test the single-track fitting result for quadrupole family errors.

Step 4. Isolated Focusing Errors - For cases where corrector calibration or quadrupole calibration errors do not produce improved fitting results, one looks for isolated focusing errors. The analysis is consists of fitting sections of the storage ring as if they were sections of a transmission line. In this case, the variables are the initial conditions of the trajectory ( $x, x^{\prime}$ for horizontal) or ( $y, y^{\prime}$ for vertical). Sections of the accelerator are tested for accuracy of the fit, and extended u' 'il a discrete 'kick' in the orbit is encountered. Simulations of the beam trajectory 'forward' and 'backward' through the suspect area are often sufficient to localize the kick. Once a candidate magnet (or accelerating cavity for linacs) is chosen as the source of error, the strength can be varied in the fit.

\subsection{Multi-track analysis}

As the name implies, multi-track analysis is an extension of the single track analysis technique. The difference is that now several rows of the response matrix are analyzed simultaneously. The solution, however, must correlate across all tracks. Although multi-track analysis is a logical extension of single track analysis, the accuracy of the solution is enhanced by the simultaneous constraints on fitting the data. Parallel to the recipe given in section 2.2, we now point out the main advantages of multi-track analysis.

Step 1. BPM Errors - With multi-track analysis it is possible to determine whether a BPM reading is in error or has a systematic problem with the linearity of the response. Using multi-track analysis, we were able to determine a pair of BPMs in SPEAR with the linearity calibration factor off by a factor of 3 in the database.

Step 2. Corrector Analysis - The correctors associated with each track (row of responsc matrix) are varied independently for each closed orbit distortion. In this case, we watch for large scatter in the corrector calibration factors, which would indicate that other moceling problems exist, probably in the quadrupole strengths. Individual corrector calibration errors are easily found.

Step 3. Quadrupole Family Errors - The power of multi-track analysis is most apparent when analyzing quadrupole family errors. In particular, one can simultaneously analyze a set of horizontal tracks, a set of vertical tracks, and a me surement of dispersion to force cross-correlation between quadrupole focusing and de-focusing in orthogonal planes. For the NSLS XRAY ring, for example, we used simultaneous analysis of four horizontal orbits, four vertical orbits, and the dispersion function, to obtain a good working model for the quadrupole family strengths and a spurious kick located where the injection line is tangent to the main ring.

Step 4. Isolated Quadrupole Errors - Multi-track analysis is also useful for locating isolated quadrupole errors where single-track analysis may not detect the error. For example, if a corrector located 180 degrees apart from a quadrupole error is excited, the beam will not move in the offending quadrupole. If many correctors at a range of phase advance are used to generate a set of trajectories through the quadrupole, then the multi-track analysis can be used to detect the error. The idea of simulating trajectories forward and backward through the error (as discussed above) applies for multi-track analysis of isolated focusing errors. With multi-track analysis, we were able to determine the location of a bend magnet with wedge components bolted on backwards in the SLC positron transmission line, and detected the presence of a wiggler magnet in SPEAR that was on when the model indicated the device was off. 


\subsection{Linearized respense matrix fiuting [2]}

The drawback of multi-track fitting is that the response matrix elements between corrector and BPMs in a storage ring are non-linear functions of quadrupole strength. The fitting algorithms must therefore be non-linear, and the analysis can take a long time to generate a solution when a fast result is desired. In SPEAR, for example, there are 30 correctors in each plane, 26 BPMs and 8 quadrupole families. Using the fitting package in RESOLVE, we have found that 8 tracks analyzed with 4 quadrupole family strengths variable is about the limit before the computation takes too long. One would like, however, to include all 60 closed orbit perturbations ( 30 horizontal, 30 vertical) and all 8 quadrupole family strengths into the calculation, and to carry through an error analysis.

One method that we developed for application to SPEAR is to linearize the response matrix about the operating point of the model. The problem then becomes a linear least-squares-fit, and a solution can be quickly found, including ertor bars.

This method for Fast Calibration of the optics model (CALIF) has been developed to automate the fitting procedure including the full set of horizontal and vertical response matrix measurements. The matrix formalism allows us to expand the set of variable quadrupole strengths, solve for corrector strength and BPM linearity calibration factors, and estimate the BPM resolutions for the measured data set. The updated optics model, including statistically correlated error bars for all fitted quantities, can then be used to predict Twiss parameters at every element in the storage ring.

The objective of the CALIF algorithm is to obtain a consistent computer model of the as-built machine based on a set of difference orbit measurements. Using a first-order perturbation approach, we seek modeling errors in the following parameters

- Quadrupole gradients

- Corrector scale factors

- BPM scale factors

- BPM resolution errors

including a comprehensive error analysis of the results. From the difference orbit measurements, we first determine the BPM-to-corrector response matrix coefficients

$$
\overline{\mathbf{C}}^{i j}=\frac{\Delta x \text { at BPiM } \mathrm{i}}{\Delta \mathrm{x}^{\prime} \text { at corrector } \mathrm{j}}
$$

which are then compared to the perturbed expression for the computer model prediction, namely,

$$
\overline{\mathrm{C}}^{\mathrm{ij}}=\mathrm{C}^{\mathrm{ij}}+\sum_{\mathrm{q}} \frac{\delta \mathrm{C}^{\mathrm{ij}}}{\delta \mathrm{K}_{\mathrm{q}}} \delta \mathrm{k}_{\mathrm{q}}
$$

where $\mathrm{C}^{i j}$ and $\frac{\delta \mathrm{C}^{\mathrm{ij}}}{\delta \mathrm{k}_{q}}$ are the computer model response matrix coefficients and their derivatives with respect to the gradient of a particular quadrupole or quadrupole family, respectively. The $\mathrm{C}^{\mathrm{ij}}$ and $\frac{\delta \mathrm{C}^{\mathrm{ij}}}{\delta \mathrm{k}_{\mathrm{q}}}$ are easily calculated with accelerator modeling codes such as COMFORT. The $\delta \mathrm{k}_{q}$ are the sought after gradient errors needed to explain the measured response matrix cocfficients $\overline{\mathbf{C}}^{\mathrm{ij}}$.

Since the solution of Eq. 2.4.2 is strongly effected by errors in the linear scale factors for both the correctors and BPMs, we augment the left hand side of Eq. 2.4 .2 by variable corrector scale factors, $x^{j}$, and BPM scale factors, $y^{i}$, to arrive at a relation among the unknown quadrupole gradient errors, corrector scales, and BPM scales ,

$$
\mathrm{C}^{\mathrm{ij}}=\mathrm{y}^{\mathrm{i}} \overline{\mathrm{C}}^{\mathrm{ij}} \mathrm{x}^{j}-\sum_{\mathrm{q}} \frac{\delta \mathrm{C}^{\mathrm{ij}}}{\delta \mathrm{k}_{q}} \delta \mathrm{k}_{\mathrm{q}}
$$

Furthermore, each $\overline{\mathrm{C}}^{\mathrm{ij}}$ has an intrinsic measurement error due to the limited resolution of the BPMs which is given by

$$
\sigma\left(\overline{\mathbf{C}}^{\mathrm{ij}}\right)=\frac{\sigma(\mathrm{BPM} \mathrm{i})}{\Delta \mathrm{x}^{\prime} \text { at correcor } \mathrm{j}} \text {. }
$$

We initially assume the same value of $\sigma$ for all BPMs. 
Now we are in a position to use Eq. 2.4.3 in a linear least squares fit either for the corrector scales $x j$ and the gradient errors $\delta \mathrm{k}_{\mathrm{q}}$ while keeping the BPM scales fixed, or for the BPM scales and the gradient errors while keeping the corrector scales fixed. In SPEAR there are 30 correctors and $26 \mathrm{BPMs}$ in $\mathrm{x}$ and $\mathrm{y}$, respectively, and 8 quadrupole families, thus yielding up to a maximum of 1560 measured $\bar{C}$ ij. Since only on the order of 70 parameters are fitted, we have a huge number of degrees of freedom which allows a careful error analysis.

Then, according to Eq. 2.4.3, a matrix "A" is constructed in which the columns are related to the fitting parameters $x^{j}$ and $\delta \mathrm{kg}$, and the rows are related to the $C \mathrm{ij}$. Each row is weighted according to its associated measurement error, given by Eq. 2.4.4. In the next step, this over determined set of linear equations is inverted. Using informal, but obvious notation, we get

$$
\left(x^{j}: \delta k_{q}\right)^{T}=\left(\frac{A^{T}}{\sigma} \frac{A}{\sigma}\right)^{-1}\left(\frac{A^{T}}{\sigma}\right)\left(\frac{C^{i j}}{\sigma}\right)
$$

where $\left(\frac{A^{T}}{\sigma} \frac{A}{\sigma}\right)^{-1}$ is the covariance matrix from which the fit errors on $\left(x j^{j}: \delta k_{q}\right)^{T}$ are deduced. The colon in Eq. 2.4.5 indicates partitioning of the corrector scale and quadrupole strength error vectors. Next, the BPM resolution errors are deduced by calculating the contribution of each BPM to the net $\chi^{2}$ for the problem. The BPM resolutions are then rescaled so that each BPM contributes equally, and the $\chi^{2}$ is forced to unity. Inconsistent (noisy) BPM are rejected at this stage. This procedure is iterated until the $\chi^{2}$ remains close to unity, which typically takes 1 to 3 iterations.

The updated solution for the corrector scales is frozen and an iterative procedure similar to the one just described is launched in order to fit the BPM scale factors $y^{i}$ and the quadrupole gradient errors.

The results of a CALIF computation to calibrate SPEAR are listed in the following table. Only a few of the horizontal correctors and BPMs are shown as examples:

\begin{tabular}{|c|c|c|c|}
\hline Quadrupole & Initial Value & Einal Value & Error $(t-1-)$ \\
\hline Q3 & $-0.9316\left(\mathrm{~m}^{-2}\right)$ & -0.9293 & $0.188 \mathrm{E}-03$ \\
\hline Q2 & $0.3700{ }^{(")}$ & 0.3713 & $0.135 \mathrm{E}-03$ \\
\hline Q1 & -0.2543 & -0.2651 & $0.843 E-03$ \\
\hline QFA & 0.7711 & 0.7701 & $0.102 \mathrm{E}-02$ \\
\hline QDA & -0.7214 & -0.7314 & $0.442 \mathrm{E}-03$ \\
\hline QFB & 0.4714 & 0.4730 & $0.538 \mathrm{E}-03$ \\
\hline $\mathrm{QF}$ & 0.4301 & 0.4266 & $0.218 \mathrm{E}-03$ \\
\hline QD & -0.6651 & -0.6685 & $0.157 \mathrm{E}-03$ \\
\hline Cerrecter $(x)$ & Inicial Value & Einal Value & Error $(+1-1)$ \\
\hline HCORR 1 & 1.0 & 0.839 & 0.023 \\
\hline 1BB2T & 1.0 & 1.051 & 0.025 \\
\hline $2 \mathrm{BB} 2 \mathrm{~T}$ & 1.0 & 1.156 & 0.029 \\
\hline $\operatorname{BPM}(x)$ & Initial Value & Final Value & Error $(+/-)$ \\
\hline WISI & 1.0 & 1.033 & 0.017 \\
\hline $1 S 2$ & 1.0 & 1.074 & 0.031 \\
\hline 253 & 1.0 & 1.037 & 0.026 \\
\hline Tune & Initial Value & Einal Value & Measurement \\
\hline$Q_{x}$ & 6.864 & 6.834 & 6.838 \\
\hline $\mathrm{Q}_{y}$ & 6.635 & 6.753 & 6.749 \\
\hline
\end{tabular}

From the table, we find that the tunes of the calibratcd model agree in both planes to within 0.004 with the measured tunes. Since the tunes were not part of the fitting procedure, this result gives us confidence in the fidelity of the calibrated model. For the quadrupole strengths, we found deviations of less than $0.01 \mathrm{~m}^{-2}$, with error bars of less than $+/$ 0.001 . The corrector scale errors were in the range of $<10$ percent, with 3 percent accuracy. These results indicate that the matrix "A" was well conditioned. 
For the BPM resolutions, we found the average vertical value of $\sigma$ was about 100 microns. In the horizontal plane, the resolutions were larger, about 200-250 microns, possibly due to the butuon geometry or longitudinal misalignments of components in SPEAR. Following the installation of new BPMS and realignment of SPEAR, we will repeat the process and compare the results. The entire process, including measurement and data analysis, takes only about two hours.

\subsection{Discussion}

In this section, we have discussed several techniques useful for calibrating the linear optics model. Each of the methods, Single-Track Analysis, Multi-Track Analysis, and Linearized Response Matrix Analysis are typically used in progression. One starts with Single-Track Analysis to detect large errors in the model, and ends with analysis of the full response matrix to yield a statistically correlated model, including error bars. It is important to note, however, that in general none of the techniques can be used independently, and the final analysis of the ring model is usually developed using all three methods. It would be incorrect, for instance, to move directly to the linear CALIF method without determining if isolated quadrupole (or higher order) errors exist in the ring. If it is found that errors do exist in the ring, then one can compute the matrix of partial derivatives with respect to a single magnet, and re-run the linear CALIF program. But if CALIF is run on data containing an isolated focusing error and instructed to find a solution based on quadrupole family errors, the solution will be incorrect.

Furthermore, if the beam trajectory is off-axis through sextupoles, quadrupole components are introduced. The final analysis of the optics model is therefore critically coupled to knowledge of the absolute orbit. As the model for the absolute orbit is improved, the optics model should be upgraded accordingly. We now direct the discussion to analysis of the absolute orbit, and the issue of determining alignment errors of the quadrupoles and BPMs. Both RESOLVE and CALIF were developed with program portability in mind so that they can be run on any storage ring with minimurr. set-up time required.

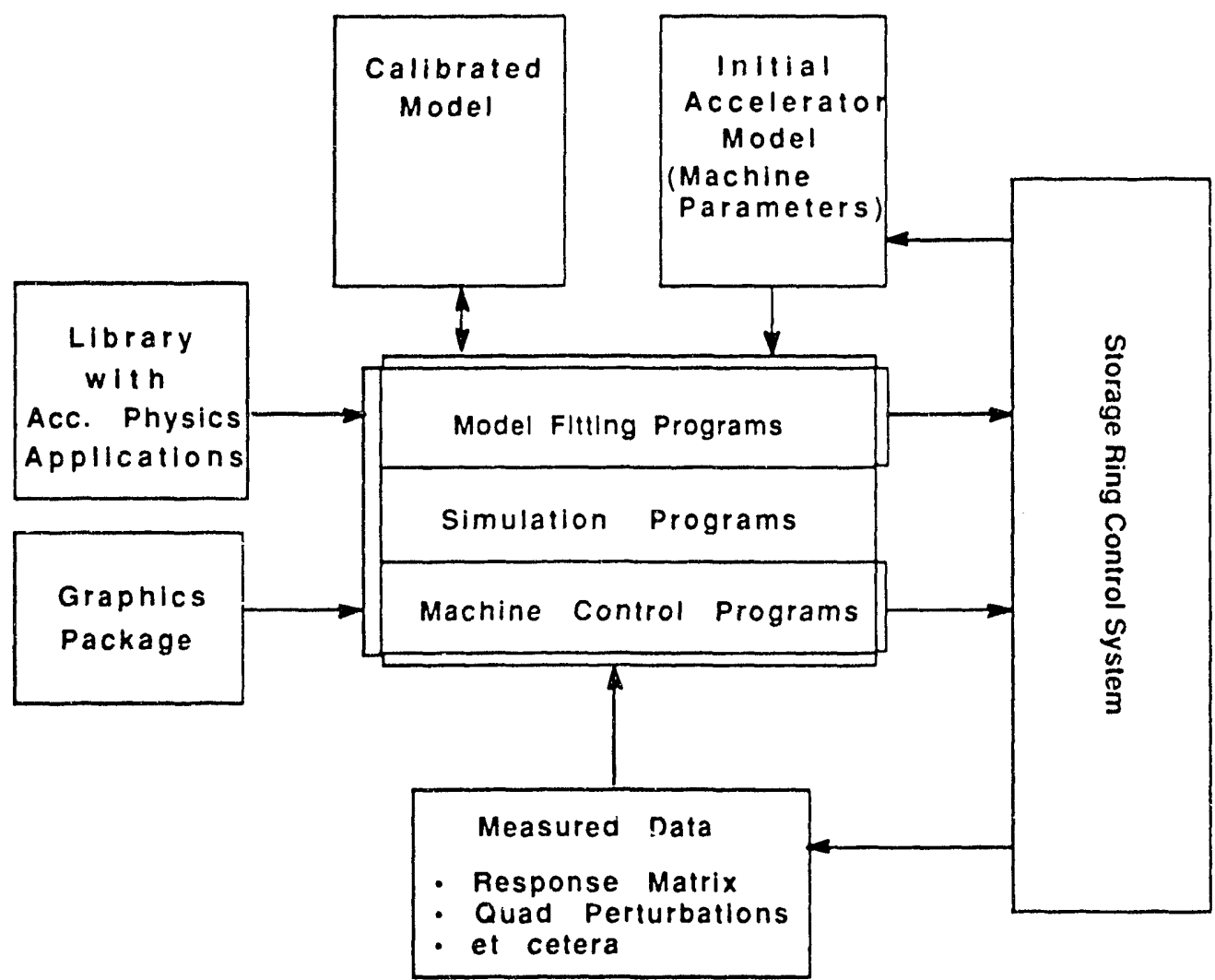

Figure 1: Schematic of Model Ralibration and Beam Control System for Storage Rings showing interaction of code library, accelerator model, and measured data with storage ring control system. Since many of these features are common to all machines, an effort should be made to standardize the organization of the interconnections for portability between laboratories 


\section{ALIGNMENT ERROR DETECTION}

Beam-based determination of component alignment in storage rings is one of the most difficult problems to analyze. In this case, no new information is obtained by kicking the beam and measuring the difference orbit since the dipole fields of offset quadrupoles generating the orbit distortion are spatially homogeneous, and BPM offset values are the same before and after the kick. Instead, the analysis typically reduces to piecing together sections of the ning which give reasonable agreement with the model based on numerically fitting trajectories through a few BPMs. These methods are outlined below. The theory can also be extended to the case where least squares fitting is used to establish a model for the alignment using the orbit measured for several different lattice configurations. This method has been pursued with success by Adolphson for the SLAC linac [3], and a formalism is outlined below for storage rings. In general, detecting all alignment errors and BPM offset values is extremely difficult. At some point the analysis must be terminated and the orbit defined in terms of the best available model for alignment errors.

\subsection{Monitor pair analysis}

One of the most simple, yet informative, techniques for orbit analysis is to consider adjacent pairs of BPM readings and interpolate the beam position in-between. Since there is a reading for the beam offset at each BPM, one simply computes the required angle at the first BPM such that the trajectory passes through the second BPM. The first order calculation must include both quadrupole focusing and dipole corrector kicks. From the SPEAR control panel, for instance, the orbit can be displayed in this way using the 'interpolate' mode. The alternative is to connect BPM readings with a straight line. A quick scan of the result graphically illustrates where there might be problems either in the readback values of absolute orbit or in the alignment of the ring. When a difference orbit is analyzed, the calculation of the interpolated beam trajectory between BPMs should be very accurate if the model is well calibrated.

For example, in SPEAR we often see the peak displacement of the absolute orbit between pairs of BPMs on the order of a few mm in most of the ring. In certain positions, however, the interpolated orbit can reach tens of mm, a clear indication of either a monitcr offset or an alignment error in the ring. To test for monitor of fset, the suspect monitor is deleted from the calculation, and the prediction for the interpolated beam trajectory skips to the next monitor. If the calculation with the suspect monitor deleted indicates a lower orbit amplitude, then we question whether the deleted monitor has an offset error. If the interpolated orbit amplitude is still large, the operator must systematically search the suspect region to see if the orbit can be interpolated between any pair of BPMs without generating an excessively large amplitude. The persistence of large amplitudes indicates the presence of a kick (eg quadrupole offset) that is not in the model of the accelerator.

The next level of sophistication in the analysis of adjacent monitor pairs is to examine the continuity of the slope of the interpolated orbit from one monitor pair to the next. If the slope of the orbit is nearly continuous, a least squares fit can be made across all three monitors. In this case, there are three constraints (beam position readings) on two or three fitting variables: initial displacement, initial angle and possibly energy offset. If the slope of the interpolated orbit is not continuous from one monitor pair to the next, then the operator must investigate the discontinuity further. Since the range of possibilities is large (any one of the three BPMs has offset, any quad in the span of the three BPMs has on offset, etc) the problem is complicated. The source of the error can be further investigated by expanding the range of BPMs under consideration.

\subsection{Monitor triplet analysis}

The first extension of interpolating the beam trajectory between adjacent pairs of BPMs is to fit the orbit for triplets of BPMs, with the first inonitor incremented by one as the analysis piogresses around the ring. A comprehensive algorithm for analyzing BPM triplets for the dispersion and beam angle at each BPM is given by A. Luccio[4]. With this technique, it is possible to distinguish between errors due to kicks (different result when two groups of three have an interval in common) or BPM offsets (different result when two groups have a monitor iil common). Dr. Luccio has successfully applied MAD to implement his algorithm, and found errors in the AGS Booster that were later confirmed to be improper electrical connections.

A similar technique to use triplets of BPM readings has been applied to SPEAR using RESOLVE. In this case, we chose three monitors and fit the initial trajectory conditions $\left(x, x^{\prime}, \eta\right)$. The quality of the fit is then used to detect regions of the ring where either BPM offset errors or alignment errors cause discrepancy between the model and measurements. The difficulty in SPEAR at this time is that the (unknown) BPM offsets are large, making it difficult to define a reference for the analysis. 


\subsection{Residue analysis}

An extension of the monitor triplet analysis that has been developed by $A$. Verdier uses the method of residues [5]. In this procedure, an automatic program is used to systematically analyze the fit of the trajectory to adjacent BPM triplets, quadruplets, quintuplets, et cetera. The 'residual' or $\chi^{2}$ for the problem is recorded for each step of the problem. A matrix of fitting residuals is compiled with the rows corresponding roughly to position in the ring, and the columns corresponding to the number of adjacent BPMs used in the fit. Using this method, the different effects due to kicks versus BPM offset errors can be distinguished (similar to section 3.2). The location of the imperfections, however, are arranged in neat diagonal arrays in the $\chi^{2}$ matrix for easy detection. This tecinnique is particularly useful for large storage rings where the amount of data makes processing difficult. In LEP, for examiple, the residual analysis routine was used detect a localized alignment error which was later corrected by moving quadrupole magnets.

\subsection{General procedure [6]} computed,

Once the model is established, the component of the closed orhit distortion (COD) induced by correctors can be

$$
\mathbf{x}_{\mathrm{C}}=\mathbf{C} \Delta \theta
$$

where $C$ is the corrector response matrix (units $\mathrm{mm} / \mathrm{mrad}$ ), $\Delta \theta$ are the corrector strengths, and $x_{c}$ is the orbit displacement evaluated at each BPM. The model can also be used predict the closed orbit distortion induced by quadrupole displacements,

$$
\mathbf{x}_{\mathrm{q}}=\mathbf{Q} \Delta \mathbf{x}_{\mathrm{q}}
$$

where $\mathbf{Q}$ has units $(\mathrm{mm} / \mathrm{mm})$ and $\Delta \mathbf{x}_{\mathrm{q}}$ is the column vector of quadrupole misalignments.

In general, the errors could be due to quadrupole angle errors, bend roll errors, etc, or sector errors where magnets are mounted to a common (misaligned) support. Note that in order to determine offset error in a particular magnet, the strength of that magnet must be varied. For this analysis, we assume the errors to emanate from of fset errors at the quadrupoles.

Superposition then yields the net closed orbit distortion $(\operatorname{cod})$ :

$$
\mathbf{x}_{\mathrm{cod}}=\mathbf{x}_{\mathrm{C}}+\mathbf{x}_{\mathrm{q}}=\mathbf{C} \Delta \theta+\mathbf{Q} \Delta \mathbf{x}_{\mathrm{q}} \text {. }
$$

Isolating the quadrupole contribution,

$$
\mathbf{Q} \Delta \mathbf{x}_{\mathrm{q}}=\mathbf{x}_{\mathrm{cod}}-\mathbf{C} \Delta \theta \text {, }
$$

one can begin to solve for the quadrupole offset vector $\Delta \mathrm{x}_{\mathrm{q}}$ using standard techniques:

1. Q-Matrix Inversion (e.g. Singular Value Decomposition

2. Most Effective Quadrupole (MICADO)

3. GOLD Method (Piecewise Solution)

In SPEAR, however, the problem is complicated by constant but unknown BPM readback errors, and an unknown energy offset of the beam. We have

$$
x_{c o d}+\Delta x_{b}=C \Delta \theta+Q \Delta x_{q}+\eta \Delta p / p
$$

where $\Delta \mathbf{x}_{\mathrm{B}}$ is the column vector of BPM readback errors and $\eta$ is the dispersion function evaluated at each BPM. In matrix form,

$$
\mathbf{x}_{\operatorname{cod}}-\mathbf{C} \Delta \theta=[-\mathbf{I}: \mathbf{Q}: \eta]\left[\Delta \mathbf{x}_{\mathrm{b}}: \Delta \mathbf{x}_{\mathrm{q}}: \Delta \mathrm{p} / \mathrm{p}\right]^{\mathrm{T}}
$$

where $\mathbf{I}$ is the identity matrix, and the colons indicate partitioning of vectors and matrices. With the set of unknowns expanded to $S=\left\{\Delta x_{b}: \Delta x_{q}: \Delta p / p\right)$ use and interpretation of techniques $i-3$ above is complicated. 
One way to find the set of unknowns $S$ is the following. By changing quadrupole strengths, we can experimentally generate a linearly independent set of Eq. 3.4.1 with different response matrix coefficients $C_{i j}$ and $Q_{i j}$, and $\eta_{i \text {, and least- }}$ squares fit the expanded set of equations to solve for the quadrupole offsets, BPM offsets and energy error.

The error bars associaied with the solution vector $S=\left\{\Delta \mathbf{x}_{b}: \Delta x_{q}: \Delta p / p\right)$ are the diagonal elements $\left[A^{T} A\right]^{-1}$ where $A$ is the response matrix

$$
A=\left[\begin{array}{ccc}
-I & Q_{1} & \eta_{1} \\
\cdots & \ldots & \ldots \\
-I & Q_{n} & \eta_{n}
\end{array}\right]
$$

Note that a set of $n$ measurements based on $n$ different lattice configurations fill the rows of $A$, that is, Eq. 3.4.1 repeated $n$ times to fill the rows of $A$.

Unfortunately, if we try to determine the entire solution vector $\mathbf{S}$ for the storage ring in one pass, the error bars are large. Three altematives are possible:

1. Compute the difference between the closed orbit equation Eq. 3.4.1 evaluated for each lattice configuration. The result is elimination of BPM offset errors from the solution vector $S$. Once the reduced solution vector $S=\left\{\Delta x_{q}: \Delta p / p\right)$ is found, computation of the BPM offset errors $\Delta x_{b}$ is straightforward.

2. Solve the set of Eqs. 3.4.1 for the ' $n$ '-configurations simultaneously in a piecewise fashion along sections of the ring, and reconstrur: the entire solution from the separate parts. The advantage is reduction of the set of variables, and more control over the fitting procedure.

3. Combination of methods 1 and 2 .

\subsection{Discussicn}

In this section, we reviewed several techniques for determining the BPM offset errors and quadrupole alignment errors in storage rings. The progression of difficulty in these techniques begins at two-BPM analysis and increases as more BPMs are included so that all the sources of error in the section of beamline must be identified consistently.

In general, the spurious kicks contributing to the orbit distortion come from all sources of alignment error in the beamline. Analysis of the true source of errors (ie quadrupole offsets vs. bend errors) is extremely complicated and probably not possible for most accelerators. But by determining the most likely locations of kicks and BPM offsets, these points can be checked for error, and a working model of the absolute beam orbit can be defined. Since there are now several computer programs available for detection of misalignments of components in storage rings, an effort should be made to develop a standard for reasing the beamline model and orbit files so that these codes can be readily dispersed to all laboratories.

\section{ORBIT CONTROL}

Many orbit control algorithms for storage rings are available which have been utilized with great success. There is one common feature of all these programs, however, they are all model dependent. The success of the orbit manipulation, whether to reduce the orbit to 'zero' reading in the BPMs, to control the position and angle of the beam at an insertion device, or to reduce coupling for optimum brightness [7] requires an accurate model of the optical components of the ring. The only way around the model dependency is to measure the corrector-to-BPM response matrix, but this method is less satisfying because it cannot be used to control the orbit and Twiss parameters at all locations in the storage ring. Some of the most popular orbit correction algorithms, RMS reduction, eigenvalue (harmonic) correction, most effective corrector control (MICADO), three-bump correction, have been applied successfully on a number of storage rings, and are discussed in the literature.

The discussion here is therefore directed toward recently developed orbit control algorithms based on Singular Value Decomposition (SVD) to obtain the inverse response matrix. In short, SVD provides two useful features for orbit control. One property is that the norm of the incremental corrector strength vector calculated for the orbit correction is minimized. Hence, the kicks applied to the beam orbit are small. The second is the ability to invert an ill-conditioned matrix, for instance, the case where more correctors are used than constraints (eg BPM readings). The solution again has the propenty of the least-norm vector, but also fits the constraints exactly where physically possible. Recently, an alternative eigen vector based orbit control technique has been introduced with properties similar to the SVD method [8]. 


\subsection{DC orbil control [9]}

At SPEAR, we applied the SVD matrix inversion method to the problem of maintaining steered photon beamlines while minimizing the corrector strengths.

In this case, we found that a 'corrector ironing' algorithm originally developed for the SLC final focus could be adapted to SPEAR. In the SLC application, the RMS excitation of correctors was reduced subject to constraint that the beam position remained constant in the sextupoles, and the beam trajectory remained constant at the collision point. Since there were typically more correctors than constraints, the technique of Singular Value Decomposition (SVD) was used to solve the under-determined linear system, exploiting the advantageous property of SVD to minimize the RMS of the solution vector which contains the corrector excitations.

In SPEAR, the problem translated into reducing corrector strengths across regions of the storage ring with 4 or 5 consecutive photon beamlines while maintaining the photon beams fixed on target. Geometrically, the constraint of constant photon beam position required the electron beam coordinates $\left(y, y^{\prime}\right)$ at the photon beam source point to satisfy $\Delta\left(y+L y^{\prime}\right)=0$, where $L$ is the distance from source point to monitor. The ' $\Delta$ '-operator indicates change before and after the skering correction.

For the SVD calculations, typically, five 'target' photon beam position monitors and two 'bump closure' constraints were used with about 12 (variable) corrector strengths to be mirimized. To optimize performance of the system, the accelerator optics model used for the corrector response calculation was derived using numerical fitting techniques to match the measured data. For this application, the virtues of the SVD solution were two-fold: (1) the solution satisfied the constraints e ztly (beams fixed on target) and (2) RMS reduction of the corrector strength vector caused the largest current decrease in the initially strongest correctors. The new corrector configuration therefore exhibits 'optimum' steering of the photon beams, leaving maximum overhead for the feedback systems to operate.

The second application of SVD in SPEAR for orbit control was to steer the beam toward zero in the BPMs. For this application, we first measured the response matrix to eliminate modelling uncertainties. Next, we chose all the correctors in the vertical plane to be variables, and all the BPMs as constraints.

\subsection{Experimental Results}

Initial tests of the 'corrector ironing' algorithm produced a 30 percent RMS reduction in the vertical corrector strengths relative to the nominal steer ad configuration. More important, after a 3 month shutdown with magnet realignment and the decision to operate SPEAR with a new high-brightness, lattice, a new corrector configuration had to be found in which all photon beam lines were steered properly. Ising the corrector-ironing program, the steering procedure was simplified to a series of closed bump corrector adjustments to position the photon beams, followed by 'ironing' the correctors to gain overhead for the next iteration. In addition to producing an optimally steered orbih it was estimated that the corrector ironing program saved about one week of accelerator commissioning time.

One of the most dramatic demonstrations of corrector strength reduction came about after rough vertical beam steering was completed. In the first iteration for the horizontal correctors, the RMS excitation of the horizontal correctors in SPEAR was reduced from 1 mrad to $30 \mu \mathrm{rad}$ and still the beams remained on target.

\subsection{Further A splications}

The extension of possible applications for the SVD-based corrector ironing program used at SPEAR ranges from generating closed-bumps to AC orbit feedback systems. Here, we list a few:

1. Commissioning Tool - A terminal interactive (X-Windows) system is planned for SPEAR which will allow the operator to adjust the electron beam orbit at selected BPMs (or photon beam position monitors) using a selected subset of corrector magnets with strengths computed via SVD. This tool will allow the operator to quickly steer photon beams on target with optimum corrector settings. In general, local beam bumps can be constructed for injection, photon beam steering, or coupling control, for example. The corrector strength weighting feature allows the user to depress individual corrector strengths.

2. Photon Beamline Alignment - By assigning the position of photon beam monitors as variables, low-strength solutions for the corrector pattern can be determined which would require realignment of the photon beamlines. Conversely, with sufficiently accurate knowledge of the photon beam position monitor elevations relative to the plane of the storage ring, SVD (or direct computation) can be used to estimate magnet misalignments. 
3. Closed Orbit Control - The original 'closed bump' SVD program for corrector ironing in SPEAR was modified for closed orbit operation. In addition, the photon beam position constraint, $\Delta(y+L y)=0$, was replaced with a new 'drif/negative-drift' model whereby the photon beam is propagated along a drift of length ' $L$ ' from the source point to the photon BPM, followed by a 'negative-drift' back to the source point. Effective phase advance, betafunctions, and response matrix coefficients can be computed at the photon BPMs.

\section{INTEGRATED SYSTEMS}

One of the most important modem aspects to model calibration and beam control systems for storage rings is to develop algorithms in a modular form so that they can be used interchangeably between laboratories. If new developments are made with this goal in mind, then all storage ring facilities share in each new advance leaving more time for conceptual design of further beam control ideas instead of re-programming standard routines. Two systems that we have been pursuing contain elements of modular interchangeably, but each would benefit from a well designed protocol making the code more transportable for use at other laboratories.

\subsection{Central library system}

In this system, a central library of accelerator analysis sub-routines is maintained which can be accessed for specific calculations (see Fig. 1). One of the main features of the architecture is that the accelerator optics model is accessible from a library routine for calculation of R-matrix parameters, closed orbit response, Twiss parameters, etc. In the case of SPEAR, the model is updated by using the linear perturbation routine described in section 2.4 , which depends on the procedures contained in the library to compute the response matrix parameters. Hence, the calibration is made with selfconsistent software. Other routines in the library include programs for varying quadrupoles while keeping tunes constant, a corrector reduction program [9], and the alignment detection software described in section 3. Recently, a fast orbit feedback simulation routine has been developed for SPEAR based on machine parameters calculated using this software.

The one common feature of all these routines is that they can be found in a central library, linked together, and access the same accelerator model. Although the accelerator model is in a quasi-transport format, a translator for MAD files has recently bein completed.

Another system that features a 'central library' format is the accelerator simulation code TRACY [10] developed for the ALS. With TRACY, each user can program a unique set of accelerator simulation commands drawing from a library of special PASCAL functions, for example, 'GetTune $\left(Q_{x}, Q_{y}\right)$ '. Some of the most advanced developments in library sysiems for accelerator simulation and control are due to Nishmura, most recently reference [11].

\subsection{Graphical interface package}

Efforts are presently underway at the SRRC light source facility in Taiwan to develop a graphical interface package modeled after RESOLVE [1], the Accelerator Interface Module (AIM) [12], and more generally, the Generic Lattice Debugger GLAD [13]. The Machine Physics Application Program (MPAP) [14] is a graphical X-Windows interface that allows the operator to fit betafunctions, manipulate the closed orbit, detect errors in the optics model, or detect accelerator alignments. The fundamental concept behind MPAP is that the operator can quickly toggle the mode of the routine to perform the indicated operations, and maintain the same 'look' to the interface. Hence, plots of the betafunctions, orbit correction, or comparisons between simulated and measured orbits are all made with the same graphics calls, and the user sees the same icons representing elements in the beamline.

The philosophy behind MPAP is twofold. First, by arranging many accelerator physics application modules in one unified code, the user can quickly perform different functions commonly used both in the control room and for off-line simulation. Each module is designed as a 'plug-in' unit that inherits the graphics software already in place. If a new piece of software is developed with the MPAP protocol, it can quickly be incorporated into MPAP. The modules are coded in ' $\mathrm{C}$ ', with the system architecture coded in $\mathrm{C}++$ to take advantage of the object-oriented features.

The second element of the MPAP philosophy lies in the connectivity to the database. In principle, to connect MPAP to each new storage ring, one only needs to supply a MAD format accelerator file and the subroutines is acquire the machine hardware parameters, power supply strengths, corrector settings, and BPM readings. 


\section{CONCLUSIONS}

In this paper we have reviewed techniques for calibrating the optics model for storage rings and for detecting errors in the alignment of the machine. For modern, high-brightness, storage rings, it is becoming increasingly more important to calibrate the optics model in order to control the beam orbit, emittance, and phase-space profile at the photon beam source points. The model is also required for the reliable development of the error detection and feedback systems needed for the new light sources.

in addition, practical orbit correction methods were discussed with the emphasis on systems which require minimum corrector strengths. By maintaining corrector strengths at the lowest possible level, parasitic sources of dispersion are minimized, and the margin of operation for fast orbit feedback systems is increased. Accurate modeling of the accelerator is also important for these applications. The need to develop a universal system for model calibration and beam control is here. At the present time, there are many laboratories that utilize similar orbit control algorithms, and are developing model calibration software. All of these routines should be implemented on a similar software platform with easily accessed and well documented 'hooks' for connecting to the database of every machine. This would permit porability of code, leading to the rapid development of a system that can be shared by laboratories around the world.

\section{CKNOWLEDGMENTS}

The authors would like to thank Max Comacchia who recognized the significance of model development early on and has encouraged progress in this direction.

\section{REEERENCES}

[1] RESOLVE is a graphical program cesigned to adjust the accelerator mode! to match measured data on an interactive basis. Some of the original ideas programmed into RESOLVE are discussed in 'Analysis of Orbit Errors in the CERN Accelerators Using Model Simulations', M. Lee, et al., Europhysics Conf. on Control Systems for Experimental Physics, Villiars, Switzerland, 1987;SLAC-PUB 4411(A). More recent applications are contained in 'Accelerator Simulation Using Computers' M.J. Lee, Y. Zambre, W. Corbett, Int. Conf. Cum Workshop on Current Trends in Data Acquisition and Control of Accelerators, Calcutta, India, 1991;SLAC-PUB-5701(A) and in 'Automatic Beamline Calibration Procedures', WJ. Corbelt, M.J. Lee and Y. Zambre, Proc. 3rd EPACS, Berlin, 24-28 March, 1992 p. 753;SLAC.-PUB-5776(A). [2] 'A Fast Model Calibration Procedure for Storage Rings', W.J. Corbeu, M.J. Lee and V. Ziemann, IEEE Particle Accelerator Conference, Washington, DC, May 17-20, 1993;SLAC-PUB-6111(A).

[3] 'Method to Evaluate Steering and Alignment Algorithms for Controlling Emittance Growth', C. Adolphson and Tor Raubenheimer, ibid.

[4] 'Ring Diagnostics and Consistency Test of the Model for the AGS Booster', A. Luccio, ibid.

[5] 'An Automatic Finder of Field Defects in a Large A.G. Machine', A. Verdier and J.C. Chappelier, ibid.

[6] 'Procedure for Determining Quadrupole and BPM Offset Values in Storage Rings', WJ. Corbeu and V. Ziemann, ibid.;SLAC-PUB-6112(A).

[7] 'Plans w Increase Source Brightness in the NSLS X-Ray Ring', J. Safranck and S. Krinsky, ibid.

[8] 'Optimization Method for Orbit Correction in Accelerators', E. Bozoki and A. Fricdrnan, ibid.

[9] 'Optimum Stecring of Photon Beamlines in SPEAR', W.J. Corbeu, B. Fon:g, M.J. Lee and V. Ziemann, ibid.;SLAC. PUB-6110(A).

[10] ' TRACY, A Tool for Accelerator Design and Analysis', H. Nishmura, LBL REPORT 25236, ESG-40, June 1988.

[11] 'Dynamic Accelerator Modelling', H. Nishmura, IEEE Particle Accelerator Conference, Washington, DC, May 17-20, 1993.

[12] 'Control System User Interface for Accelerator Commissioning and Operation', D. Dobroth, et al, IEEE Particle Accelerator Conference, San Francisco, CA, 1991, p. 1305.

[13] 'GLAD: A Generic Lattice P: öugger', M.J. Lee, Int. Conf. on Accelerators and Large Experimental Physics Control Systems, Tsukuba, Japan, 1991;SiAC-PUB-5700(A).

[14] 'Machine Physics Application Program for Control, Commissioning and Error Findings for Storage Rings', H.P. Chang, et al, IEEE Particle Accelerator Conference, Washington, DC, May 17-20, 1993. 

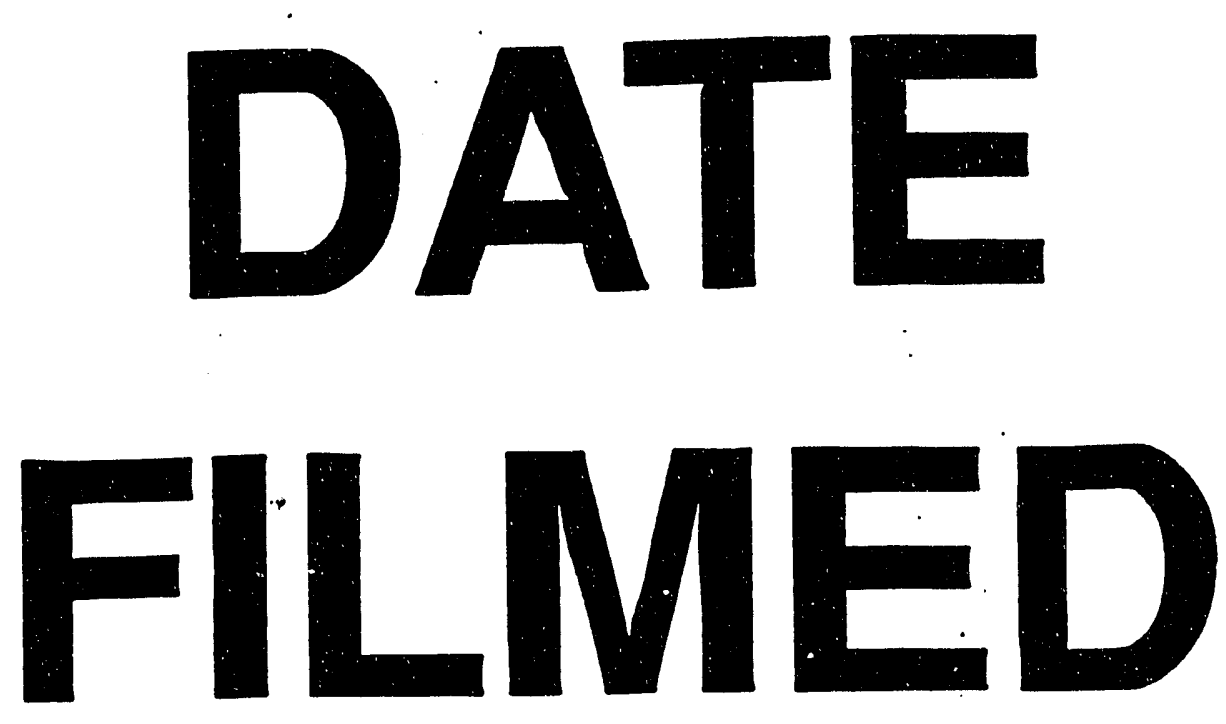

$12 / 8 / 93$
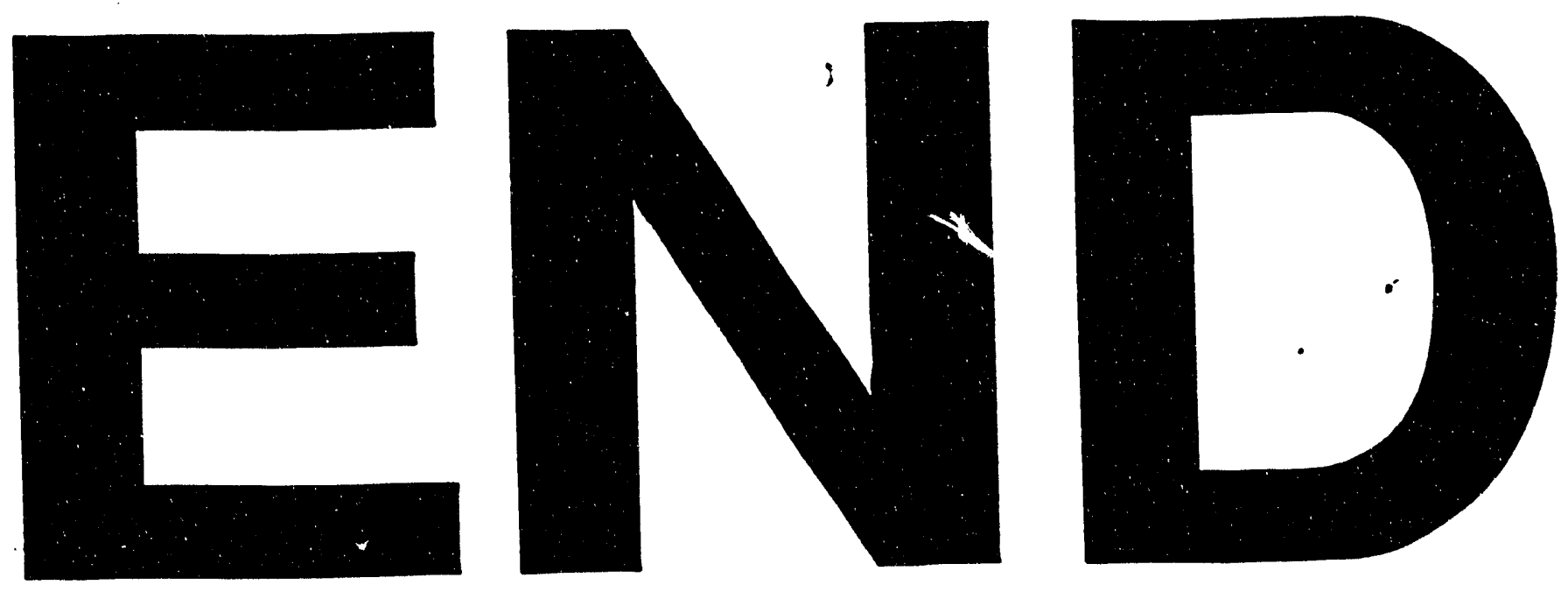
